\title{
GMR
}

\section{Letter to the Editor \\ Partial diallel crosses for predicting yield of semiexotic maize populations}

\author{
C.M. Silva ${ }^{1}$, J.B. Miranda Filho ${ }^{2}$, U.C. Mendes ${ }^{3}$ and E.F. Reis ${ }^{1}$ \\ ${ }^{1}$ Programa de Pós-Graduação em Agronomia, Universidade Federal de Goiás, \\ Jataí, GO, Brasil \\ ${ }^{2}$ Departamento de Genética, Escola Superior de Agricultura Luiz de Queiroz, \\ Universidade de São Paulo, Piracicaba, SP, Brasil \\ ${ }^{3}$ Programa de Pós-Graduação em Agronomia, Universidade Estadual Paulista, \\ Campus Ilha Solteira, SP, Brasil \\ Corresponding author: E.F. Reis \\ E-mail: esesiofr7@gmail.com
}

Genet. Mol. Res. 16 (1): gmr16019544

Received November 21, 2016

Accepted January 23, 2017

Published February 8, 2017

DOI http://dx.doi.org/10.4238/gmr16019544

Copyright $(2017$ The Authors. This is an open-access article distributed under the terms of the Creative Commons Attribution ShareAlike (CC BY-SA) 4.0 License.

\begin{abstract}
In Brazil, the total production of maize has increased continuously due to the advances in technology but also to the new frontiers for the maize crop, including the expansion of the second crop (safrinha; usually maize after soybean). Therefore, the exploitation of new sources of germplasm seems to be imperative to attend the actual and future demands for modern cultivars adapted to the greatly variable environments available to the maize crop. The present study was based on a partial diallel (intergroup) cross to provide quantitative information on the potential of two groups of varieties (NAP - exotics and HG - local) and their combining abilities to direct the synthesis of new populations to be used in hybrid crosses or as a base for breeding programs. The experiments were carried out in two planting seasons (safra - 1st crop; safrinha - 2nd crop) following the completely randomized block design with four replications of plots $5 \mathrm{~m}$ long spaced $0.9 \mathrm{~m}$ with 25 plants per
\end{abstract}

Genetics and Molecular Research 16 (1): gmr16019544 
plot after thinning. The following traits were analyzed: male flowering (days), stalk diameter (mm), plant height (m), ear height (m), tassel length $(\mathrm{cm})$, tassel branch number, ear length $(\mathrm{cm})$, ear diameter $(\mathrm{cm})$, ear yield ( $\mathrm{t} / \mathrm{ha})$, and grain yield $(\mathrm{t} / \mathrm{ha})$. The populations under study presented acceptable levels for yield potential and agronomic traits, thus assuring good perspectives for their use in breeding programs. The prediction for the new populations to be synthesized from the cross between the two groups of varieties contributed positively and HG-3 population was the most appropriate, in both eras, as the parent variety most promising in Group II (HG to be used as a base) for incorporation of varieties of Group I (NAP).

Key words: Diallel analysis; Genetical enhancement; Prediction of composites

\section{INTRODUCTION}

The maize crop has been recognized as one of the most important for the expansion of the Brazilian agriculture. In the last three decades, maize has been cultivated in two seasons of the same year: 1st crop (safra - October to January) and 2nd crop (safrinha - February to May), with planting date varying in different regions. The yield level of the 2 nd crop (usually corn after soybean) has increased continuously during the last years and in some regions the cultivated area has been higher than the 1st crop. The Central West region represents more than $65 \%$ of the maize crop in the 2nd season in Brazil (CONAB, 2015). Besides the expansion of the maize crop in time it also has occurred extensively in space by opening new frontiers, thus increasing the variation of environments (latitude, altitude, fertility, biotic and non-biotic stresses, etc.). Such great changes justify the interest for new sources of germplasm to increase the chances of success of breeding programs to attend the new and expanding demands for adapted and outstanding genotypes.

The introduction of exotic germplasm in breeding programs has long been recognized as an important strategy to increase genetic variability and for incorporation of important genes not existing in the local varieties (Goodman, 1985; Nass et al., 2001). In Brazil, the great variability that characterize the maize germplasm has a significant contribution of exotic sources that largely contributed to increase the efficiency of breeding programs for the development of cultivars (Miranda Filho, 1992; Santos and Miranda Filho, 1992; Regitano Neto et al., 1997). Old local varieties such as Cateto and Dente Paulista were the first sources for the development of the hybrid seeds. The introduction of exotic germplasm, mainly Tuxpeño and related races of Mexico and Central America, largely contributed for the development of outstanding semident hybrids (Miranda Filho and Viégas, 1987). Later on, several other introductions were continuously accomplished (Moro et al., 1981; Nass et al., 2001). In spite of the cognizance on the role of previous introductions, recently there has been an increasing interest for new sources of germplasm, particularly focusing the resistance or tolerance to several kinds of stresses (Basso and Miranda Filho, 2001; Oliveira et al., 2015).

The present study focused in a first plane the evaluation of crosses between two groups of germplasm (I. exotic; II. local) to get knowledge on their intrinsic genetic properties in order to direct actions for incorporation and development of new populations to be used in breeding programs.

Genetics and Molecular Research 16 (1): gmr16019544 


\section{MATERIAL AND METHODS}

Two groups (I and II) of open-pollinated populations (herein designated as varieties) were used in the present study based on crosses between groups, following the partial diallel scheme as given by Geraldi and Miranda Filho (1988).

\section{Origin of Group I}

The project named as Núcleo de Apoio à Pesquisa do Milho (NAP-MILHO) was coordinated by the Department of Genetics (ESALQ-USP, Piracicaba, SP, Brazil) and conducted in a cooperative system involving several public and private research institutions for the identification of sources of resistance for leaf diseases among accesses of the Brazilian Germplasm Bank of Maize (CENARGEN-EMBRAPA). The selection process led to the synthesis of five populations (composites) that were symbolized by NAP and related to specific foliar diseases, as follows: NAP-PP, NAP-PM, NAP-PZ, NAP-HT, and NAP-CS, representing resistance to Puccinia polysora, Phaeosphaeria maydis, Physopella zeae, Exerohilum turcicum, and corn stunt complex, respectively. Other four populations were later derived from the selected accesses after phenotypic selection for type of endosperm, and were designated as NAP-FA, NAP-FL, NAP-FB, and NAP-DB, with characteristics recognized as yellow flint, orange flint, white flint, and white dent, respectively. Therefore, the nine NAP populations comprised the Group I as already mentioned. More details about the origin of the NAP populations are given by Miranda Filho et al. (2000) and Mendes et al. (2015).

\section{Origin of Group II}

In the period covered by the years 2000 to 2004, several samples of commercial maize crops were collected in the field of local farmers within the region of the Southwest of Goiás State; such samples represented the $F_{2}$ generations of maize hybrids with acceptable patterns of productivity, adaptation and important agronomic traits such as lodging resistance, plant architecture and tolerance to biotic and non-biotic stresses. The sampled populations were symbolized as HG and five of them were chosen to compose Group II of varieties used in the present study.

The two groups of populations (varieties) were identified by numbers as follows: Group I: [1. NAP-FA, 2. NAP-FL, 3. NAP-FB, 4. NAP-DB, 5. NAP-HT, 6. NAP-PP, 7. NAPPZ, 8. NAP-PM, 9. NAP-CS]. Group II: [1'. HG-40, 2'. HG-54, 3'. HG-55, 4'. HG-75, 5'. HG-81]. The 14 parental varieties were multiplied by sib-cross within varieties and crossed according to the partial diallel (intergroup) as suggested by Geraldi and Miranda Filho (1988). Therefore, the whole set of entries (59) in the diallel scheme comprised 14 parental varieties and 45 hybrid crosses. The commercial hybrids SH4080 and BG7046 were used as checks in the 1st and 2nd crops, respectively.

The yield trials were conducted in the period of 2013/2014 at UFG (Universidade Federal de Goiás, Regional Jataí, GO, Brazil), as randomized complete blocks with four replications of plots $5 \mathrm{~m}$ long spaced $0.90 \mathrm{~m}$ apart with 25 plants per plot after thinning. The following traits were analyzed: MF - male flowering (days), SD - stalk diameter (mm), PH - plant height (m), EH - ear height (m), TL - tassel length (cm), NB - tassel branch number, EL - ear length $(\mathrm{cm})$, ED - ear diameter $(\mathrm{cm}), \mathrm{EY}$ - ear yield $(\mathrm{t} / \mathrm{ha})$, and GY - grain yield $(\mathrm{t} / \mathrm{ha})$. The preliminary analysis of variance followed the model:

Genetics and Molecular Research 16 (1): gmr16019544 


$$
Y_{i j}=\mu+t_{i}+b_{j}+e_{i j}
$$

(Equation 1)

where $Y_{i j}$ is the plot expression of the $\mathrm{i}^{\text {th }}$ treatment at the $\mathrm{j}^{\text {th }}$ replication whose effects are represented by $t_{i}$ (fixed) and $b_{j}$ (random); $e_{i j}$ is the random error term for individual plots. In the analysis, the experimental unity was represented by plot totals (MF, EY, GY) or plot means (SD, PH, EH, TL, NB, EL, ED: means of five plants or ears per plot).

Analysis of the variance of the diallel table (parental varieties and crosses) was accomplished with plot means of four replications, using the model presented by Geraldi and Miranda Filho (1988). In such model, the effect of treatments is partitioned in three groups: Varieties I, Varieties II and hybrids (variety crosses), representing Group I, Group II and hybrids (Group I x Group II). The diallel itself is analyzed only with variety crosses and the appropriate model is an adaptation of Method-IV of Griffing (1956), as shown by Geraldi and Miranda Filho (1988):

$$
Y_{i j}=\mu+g_{i}+g_{j}+s_{i j}+\bar{e}_{i j}
$$

where $\bar{e}_{i j}$ is the error term adjusted to means of $\mathrm{r}$ replications, or

$$
e_{i j}=\sum_{j} e_{i j} / r
$$

$\mu$ is the mean of all crosses, $g_{i}$ and $g_{j}$ are the effects of general combining ability (GCA) of varieties in Group I and Group II and $s_{\mathrm{ij}}$ is the effect of specific combining ability (SCA); except for $\bar{e}_{i j}$, all other effects are fixed. An alternative model for the analysis of the diallel table should be the model for partial diallel suggested by Miranda Filho and Geraldi (1984), that is based on the heterosis and its components, as an adaptation to the model of Gardner and Eberhart (1966); however, for the purpose of the present study the model discussed by Geraldi and Miranda Filho (1988) was considered the most appropriate.

As already mentioned, the main purpose of the present study was to obtain information about important quantitative characters in two distinct groups of populations (herein designated as varieties) and their use for guiding the synthesis of new populations for breeding objectives.

The methodology for prediction of population means was used for the identification of two types of populations: a) Population resulting from the recombination of the variety cross (NAP $x$ HG = Group I $x$ Group II), for the identification of the local population (HG from Group II) most promising for the incorporation of each semiexotic population from Group I. b) Hybrid combinations (NAP x HG) most appropriate for the exploitation of heterosis at the interpopulation level, also looking forward to the development of inbred lines to be used in hybrid crosses.

In (a) it was used the prediction formula given by Miranda Filho (1974) and Hallauer and Miranda Filho (1988):

$$
\varphi=\frac{1}{2}\left(\bar{Y}_{0}+\bar{Y}_{v}\right)+\frac{1}{2} \bar{h}_{0}+\frac{n-1}{4 n} \bar{h}_{v}
$$

where $\varphi$ is the expected mean of the composite formed with $50 \%$ of one semiexotic variety

Genetics and Molecular Research 16 (1): gmr16019544 
(NAP, Group I) and 50\% of varieties of Group II (HG), used as basic material for incorporation; $\bar{Y}_{0}$ is the observed mean of the semiexotic population; $\bar{Y}_{V}$ is the mean of k varieties used as the base for incorporation; $\bar{h}_{0}$ is the average heterosis of crosses between the semiexotic variety and the k varieties of Group II; $\bar{h}_{V}$ is the average heterosis of crosses between the k varieties. In the present study, only one variety HG will be used for incorporation of each NAP, so that $\mathrm{k}=1, \bar{h}_{V}$ does not exist and the prediction formula and is reduced to

$$
\varphi=\frac{1}{2}\left(\bar{Y}_{0}+\bar{Y}_{v}\right)+\frac{1}{2} \bar{h}_{0}
$$

which is equivalent to the formula of Mather and Jinks (1982) for prediction of the $\mathrm{F}_{2}$ generation from the cross between two inbred parents. In the present case, the resulting population is a composite of size $\mathrm{s}=2$, obtained by recombination of the $\mathrm{F}_{1}$ hybrid between two varieties. Since the recombination is a random procedure, the newly formed composite will be in HardyWeinberg equilibrium and literally ready to initiate a recurrent selection program.

In the scheme (b), the interest is on the hybrid between two composites, one from each group (I and II). For that purpose, selection of the basic germplasm was done in two distinct phases:

$\left(b_{1}\right)$ - Selection of the most promising varieties based on the effects of GCA in each group ( $\mathrm{g}_{\mathrm{i}}$ in Group I and $\mathrm{g}_{\mathrm{j}}$ in Group II), estimated according to the model for partial diallel shown by Geraldi and Miranda Filho (1988); thus, only the varieties with positive estimates of $g_{i}$ and $g_{j}$ were chosen for predicting means, as planned. Finally, the following sets of varieties were chosen: $(3,6,7,8)$ in Group I and $\left(4^{\prime}, 5^{\prime}\right)$ in Group II, under the conditions of 1st crop; and $(1,6,7,8)$ and $\left(3^{\prime}, 5^{\prime}\right)$ under the conditions of 2 nd crop, respectively.

$\left(b_{2}\right)$ - Predicting means of hybrids between composites of different groups. With the $n_{1}$ varieties taken from Group $\mathrm{I}, \mathrm{n}_{\mathrm{p} 1}=2^{\mathrm{n} 1}-1$ populations (combinations) will be available, including the $\mathrm{n}_{1}$ varieties plus $2^{\mathrm{n} 1}-\left(\mathrm{n}_{1}+1\right)$ composites of size $\mathrm{k} \geq 2$; for $\mathrm{n}_{1}=4$, it follows $\mathrm{n}_{\mathrm{p} 1}=15$. In Group II, it will be available $n_{p 2}=3$ (two varieties and the composite of size $\mathrm{s}=2$ ). Therefore, the possible number of hybrids between composites will be $\mathrm{N}_{\mathrm{c}}=\mathrm{n}_{\mathrm{p} 1} \cdot \mathrm{n}_{\mathrm{p} 2}=45$. The same calculation holds for both 1st and 2nd crops because the same number of available varieties. The predicted mean of the hybrid between composites (composite I x composite II) is given by

$$
\varphi_{\mathrm{I}} \times \varphi_{\mathrm{II}}=\left(\sum_{i}^{k 1} f_{i} V_{i}\right)\left(\sum_{j}^{k 2} f_{j} V_{j}\right)
$$

(Equation 6)

where $f_{i}$ is the proportion that each variety of Group I $\left(V_{i}\right)$ enter in the composite; the same thought applies to varieties of Group II $\left(\mathrm{V}_{\mathrm{j}}\right)$. Actually, the formula above is symbolic and if expressed algebraically would be

$$
\varphi_{\mathrm{I}} x \varphi_{\mathrm{II}}=\Sigma_{i, j} f_{i} f_{j} V_{i} V_{j}
$$

which still is a conceptual definition, that to be an effective formula the product $\mathrm{V}_{\mathrm{i}} \mathrm{V}_{\mathrm{j}}$ must be replaced by the observed mean of the hybrid $\mathrm{H}_{\mathrm{ij}}$; then, the effective formula for prediction of the cross between two composites (Eberhart et al., 1967; Vencovsky and Barriga, 1992) is

Genetics and Molecular Research 16 (1): gmr16019544 


$$
\varphi_{\mathrm{I}} x \varphi_{\mathrm{II}}=\Sigma_{i, j} f_{i} f_{j} H_{i j}
$$

(Equation 8)

In the present case, the representation of the genetic source within brackets may include only one variety with frequency $\mathrm{f}_{\mathrm{i}}=1$, as in the example $\left[\mathrm{V}_{1}\right] \times\left[\frac{1}{2} V_{\mathrm{I}^{\prime}}+\frac{1}{2} V_{2^{\prime}}\right]$, which would result in the hybrid with mean predicted by $\frac{1}{2}\left(\mathrm{H}_{11},+\mathrm{H}_{12},\right)$.

In the present study, with previous selection of varieties based on estimates of GCA, the prediction process reduces to 45 crosses, as already shown. Nevertheless, the prediction of all possible hybrids comprising the whole set of varieties (9 in Group I and 5 in Group II) would lead that number to $\left(2^{9}-1\right)\left(2^{5}-1\right)=15,841$.

\section{RESULTS AND DISCUSSION}

\section{Means and variances}

The observed means of ten plant and ear traits are shown in Tables 1 and 2 referring to two planting seasons (1st crop - $\mathrm{e}_{1}$ : safra; 2 nd crop - $\mathrm{e}_{2}$ : safrinha, respectively); the whole set of means includes nine varieties of Group I, five varieties of Group II and 45 hybrid crosses (Group I x Group II). Means for each trait in experiments of two seasons are summarized in Table 3.

For male flowering, practically there was no difference among groups (Varieties I, Varieties II, Hybrids) with means around 59 days in $\varepsilon_{1}$ and around 60 days in $\varepsilon_{2}$. However, the earliest (lower mean) in $\varepsilon_{1}$ was for 5 (NAP-HT) in Group 1, 4' (HG-75) in Group II and the hybrid $4 \times 1$ ' (NAP-DB x HG-40); in $\varepsilon_{2}$ the earliest varieties were the same as in $\varepsilon_{1}$ and the hybrid crosses were very similar (around 60 days). It was concluded that expression of male flowering for both groups of parent varieties and hybrids was within the pattern of cultivars of maize commonly in use. Souza (2015) reported male flowering (days) as 60.9, 63.1 and 61.7 in the semi-exotic populations CRE-01, CRE-02, and CRE-03, which were higher than the check hybrids (P30K75 and SHS 4080) by 4.2, 6.0, and 4.8\%, respectively.

SD also showed to be very similar among groups, in the range of 24 to $26 \mathrm{~mm}$ in the whole set. The only difference was that means were a little lower for all groups (less than $1.1 \mathrm{~mm}$ on average) in $\varepsilon_{2}$. The same pattern of expression among groups in two seasons was observed for plant height and ear height. However, it was possible to identify 3 (NAP-FB) and 4' (HG-75) as the smaller means for plant height in $\mathrm{e}_{1}$ and 3 and $1^{\prime}(\mathrm{HG}-40)$ in $\varepsilon_{2}$. The lower means for ear height were exhibited by the same parental varieties as for plant height in both seasons.

$\mathrm{TL}$ and NB, on the average for each group, were in the range of 34 to 40 and 9 to 18 , respectively; for both traits there was a slight decrease from $\varepsilon_{1}$ to $\varepsilon_{2}$. Such ranges were somewhat below the ranges (36.1 to 46.0 and 12.6 to 23.8 , averaging 40.9 and 18.4 , respectively) reported by Andrade and Miranda Filho (2008) for half-sib family means of the maize population, ESALQ-PB1. Souza (2015) reported means for TL and NB, around 38 and 14 in three semi-exotic populations (CRE-01, CRE-02, CRE-03). For both TL and NB, as a measure of tassel size, Group I showed a slightly higher mean than Group II in both seasons, which is a coherent result, because modern germplasm derived from commercial cultivars, as is the case of varieties of Group II, tends to exhibit smaller tassels.

EL and ED showed a similar pattern of expression as for TL and NB in the three groups, i.e., Group II $<$ Hybrids $<$ Group I in $\varepsilon_{1}$ and Group II $<$ Group I $<$ Hybrids in $\varepsilon_{2}$. In general, there was a decrease in means from $\varepsilon_{1}$ to $\varepsilon_{2}$ in both traits.

Genetics and Molecular Research 16 (1): gmr16019544 


\begin{tabular}{|c|c|c|c|c|c|c|c|c|c|c|c|c|}
\hline \multirow[t]{2}{*}{$\mathrm{iij}$} & $1^{\prime}$ & $2^{\prime}$ & $3^{\prime}$ & $4^{\prime}$ & $5^{\prime}$ & $\mathrm{Y}_{\mathrm{ii}}$ & $1^{\prime}$ & $2^{\prime}$ & $3^{\prime}$ & $4^{\prime}$ & $5^{\prime}$ & $\mathrm{Y}_{\mathrm{ii}}$ \\
\hline & \multicolumn{6}{|c|}{ MF - Male flowering (days) } & \multicolumn{6}{|c|}{ SD - Stalk diameter $(\mathrm{mm})$} \\
\hline 1 & 56.8 & 59.0 & 59.8 & 59.3 & 59.0 & 59.3 & 27.4 & 26.7 & 24.7 & 24.1 & 26.8 & 26.7 \\
\hline 2 & 58.3 & 58.8 & 59.8 & 60.0 & 59.0 & 58.8 & 25.1 & 25.9 & 24.8 & 25.3 & 26.8 & 25.6 \\
\hline 3 & 57.8 & 57.8 & 60.3 & 59.5 & 59.5 & 58.3 & 25.3 & 26.0 & 24.9 & 24.6 & 27.0 & 25.0 \\
\hline 4 & 56.5 & 58.5 & 60.0 & 59.5 & 59.8 & 59.5 & 26.9 & 26.1 & 24.8 & 24.0 & 27.7 & 25.9 \\
\hline 5 & 57.5 & 56.8 & 58.8 & 57.0 & 58.8 & 57.3 & 25.1 & 25.0 & 25.5 & 24.3 & 26.4 & 26.1 \\
\hline 6 & 57.3 & 59.8 & 60.5 & 59.3 & 59.8 & 59.8 & 25.7 & 26.2 & 25.5 & 24.8 & 26.2 & 24.8 \\
\hline 7 & 58.3 & 59.3 & 60.5 & 58.0 & 59.3 & 59.3 & 26.0 & 25.6 & 26.0 & 26.5 & 25.2 & 24.2 \\
\hline 8 & 60.0 & 59.3 & 59.5 & 59.0 & 59.8 & 59.8 & 25.5 & 24.5 & 26.1 & 25.6 & 26.6 & 25.5 \\
\hline 9 & 60.0 & 59.8 & 60.0 & 59.3 & 60.0 & 59.0 & 28.1 & 24.9 & 25.2 & 26.7 & 27.0 & 25.4 \\
\hline \multirow[t]{2}{*}{$\mathrm{Y}_{\mathrm{ij}}$} & 59.3 & 59.3 & 59.8 & 56.5 & 59.8 & $57.0^{\tau}$ & 25.6 & 25.6 & 25.7 & 24.9 & 26.0 & $26.1^{\tau}$ \\
\hline & \multicolumn{6}{|c|}{ PH - Plant height (m) } & \multicolumn{6}{|c|}{ EH - Ear height (m) } \\
\hline 1 & 2.25 & 2.33 & 2.14 & 2.15 & 2.38 & 2.54 & 1.24 & 1.24 & 1.14 & 1.23 & 1.38 & 1.48 \\
\hline 2 & 2.20 & 2.37 & 2.26 & 2.38 & 2.33 & 2.47 & 1.22 & 1.42 & 1.25 & 1.40 & 1.44 & 1.47 \\
\hline 3 & 2.23 & 2.36 & 2.31 & 2.21 & 2.41 & 2.42 & 1.23 & 1.35 & 1.35 & 1.27 & 1.40 & 1.41 \\
\hline 4 & 2.14 & 2.29 & 2.36 & 2.35 & 2.45 & 2.53 & 1.18 & 1.27 & 1.38 & 1.44 & 1.44 & 1.52 \\
\hline 5 & 2.18 & 2.21 & 2.32 & 2.14 & 2.34 & 2.45 & 1.22 & 1.26 & 1.34 & 1.18 & 1.34 & 1.53 \\
\hline 6 & 2.31 & 2.53 & 2.40 & 2.46 & 2.58 & 2.61 & 1.30 & 1.55 & 1.42 & 1.48 & 1.61 & 1.67 \\
\hline 7 & 2.17 & 2.38 & 2.50 & 2.35 & 2.35 & 2.45 & 1.15 & 1.40 & 1.46 & 1.35 & 1.32 & 1.51 \\
\hline 8 & 2.41 & 2.32 & 2.42 & 2.51 & 2.41 & 2.63 & 1.41 & 1.30 & 1.43 & 1.59 & 1.45 & 1.66 \\
\hline 9 & 2.30 & 2.34 & 2.33 & 2.25 & 2.45 & 2.65 & 1.25 & 1.41 & 1.43 & 1.31 & 1.46 & 1.73 \\
\hline \multirow[t]{2}{*}{$\mathrm{Y}_{\mathrm{ij}}$} & 1.85 & 1.94 & 2.01 & 1.75 & 1.86 & $2.07^{\tau}$ & 0.99 & 1.00 & 1.13 & 0.90 & 0.98 & $1.16^{\tau}$ \\
\hline & \multicolumn{6}{|c|}{ TL - Tassel length $(\mathrm{cm})$} & \multicolumn{6}{|c|}{ NB - Tassel branch number } \\
\hline 1 & 38.5 & 39.3 & 36.4 & 38.4 & 40.4 & 39.1 & 12.6 & 14.4 & 12.5 & 13.7 & 11.3 & 16.2 \\
\hline 2 & 39.1 & 36.4 & 38.1 & 38.6 & 39.8 & 38.4 & 11.4 & 13.0 & 13.9 & 13.3 & 13.2 & 15.3 \\
\hline 3 & 40.3 & 37.6 & 37.7 & 40.4 & 41.1 & 40.0 & 13.9 & 14.9 & 14.3 & 14.0 & 13.0 & 15.7 \\
\hline 4 & 38.6 & 39.7 & 37.6 & 39.1 & 40.3 & 40.7 & 13.7 & 15.9 & 15.1 & 11.6 & 12.1 & 17.3 \\
\hline 5 & 37.9 & 37.4 & 38.9 & 37.2 & 37.9 & 37.7 & 13.5 & 16.2 & 15.1 & 15.1 & 15.2 & 18.7 \\
\hline 6 & 38.7 & 36.7 & 40.1 & 40.7 & 40.1 & 40.0 & 13.5 & 16.4 & 16.6 & 15.5 & 13.4 & 17.9 \\
\hline 7 & 40.3 & 41.0 & 39.4 & 39.2 & 37.6 & 40.0 & 14.1 & 15.1 & 14.6 & 16.5 & 11.9 & 17.9 \\
\hline 8 & 39.1 & 37.1 & 38.8 & 40.6 & 40.0 & 40.0 & 11.8 & 15.6 & 15.7 & 14.4 & 13.5 & 15.4 \\
\hline 9 & 38.4 & 37.2 & 36.5 & 40.1 & 38.0 & 38.8 & 14.9 & 15.9 & 16.7 & 14.7 & 10.9 & 20.6 \\
\hline \multirow[t]{2}{*}{$\overline{Y_{i j}}$} & 36.4 & 36.6 & 35.1 & 37.7 & 36.5 & $38.2^{\tau}$ & 10.6 & 11.9 & 12.3 & 10.4 & 8.1 & $12.0^{\tau}$ \\
\hline & \multicolumn{6}{|c|}{ EL - Ear length $(\mathrm{cm})$} & \multicolumn{6}{|c|}{ ED - Ear diameter $(\mathrm{cm})$} \\
\hline 1 & 18.4 & 17.2 & 16.7 & 17.1 & 17.1 & 17.5 & 4.80 & 4.85 & 4.75 & 4.65 & 4.90 & 4.75 \\
\hline 2 & 16.9 & 15.2 & 16.8 & 16.9 & 17.7 & 17.2 & 4.70 & 4.80 & 4.70 & 4.75 & 4.90 & 4.65 \\
\hline 3 & 16.6 & 15.7 & 17.0 & 16.4 & 17.7 & 16.7 & 4.70 & 4.65 & 4.55 & 4.65 & 4.75 & 4.50 \\
\hline 4 & 17.1 & 16.5 & 16.6 & 16.8 & 17.0 & 17.1 & 4.55 & 4.80 & 4.60 & 4.60 & 4.80 & 4.75 \\
\hline 5 & 16.5 & 16.6 & 15.9 & 16.2 & 17.6 & 16.1 & 4.45 & 4.40 & 4.80 & 4.65 & 4.85 & 4.15 \\
\hline 6 & 16.9 & 16.4 & 17.1 & 17.5 & 16.7 & 18.0 & 4.70 & 4.75 & 4.70 & 4.75 & 4.95 & 4.75 \\
\hline 7 & 17.6 & 16.5 & 17.2 & 17.4 & 16.2 & 17.0 & 4.55 & 4.90 & 4.70 & 4.75 & 4.75 & 4.45 \\
\hline 8 & 17.8 & 16.5 & 16.7 & 17.4 & 16.5 & 16.9 & 4.75 & 4.70 & 4.50 & 4.85 & 4.85 & 4.50 \\
\hline 9 & 18.2 & 16.1 & 15.1 & 17.6 & 18.4 & 16.4 & 4.70 & 4.70 & 4.65 & 4.50 & 4.85 & 4.45 \\
\hline \multirow[t]{2}{*}{$\mathrm{Y}_{\mathrm{ij}}$} & 15.1 & 15.5 & 14.9 & 17.5 & 13.7 & $17.6^{\tau}$ & 4.45 & 4.60 & 4.50 & 4.55 & 4.67 & $4.78^{\tau}$ \\
\hline & \multicolumn{6}{|c|}{ EY - Ear yield (t/ha) } & \multicolumn{6}{|c|}{ GY - Grain yield (t/ha) } \\
\hline 1 & 8.69 & 8.63 & 8.26 & 9.54 & 9.98 & 8.81 & 6.94 & 7.44 & 6.98 & 7.89 & 8.47 & 7.34 \\
\hline 2 & 9.48 & 8.92 & 8.18 & 8.45 & 9.78 & 8.37 & 8.06 & 7.66 & 6.97 & 7.17 & 8.20 & 7.12 \\
\hline 3 & 8.69 & 8.62 & 9.36 & 9.14 & 9.86 & 8.00 & 7.40 & 7.31 & 8.01 & 7.61 & 8.43 & 6.68 \\
\hline 4 & 8.98 & 9.09 & 8.30 & 9.45 & 9.03 & 8.83 & 7.64 & 7.95 & 6.66 & 8.01 & 7.71 & 7.43 \\
\hline 5 & 7.73 & 7.82 & 8.94 & 8.29 & 8.84 & 7.15 & 6.58 & 6.79 & 7.55 & 6.92 & 7.57 & 5.95 \\
\hline 6 & 9.50 & 8.91 & 9.09 & 9.31 & 9.99 & 8.48 & 7.97 & 7.65 & 7.73 & 7.93 & 8.43 & 7.11 \\
\hline 7 & 8.26 & 8.35 & 10.29 & 9.35 & 8.32 & 8.00 & 7.06 & 7.09 & 8.86 & 7.92 & 7.39 & 6.64 \\
\hline 8 & 9.30 & 8.85 & 9.30 & 10.18 & 9.36 & 7.78 & 7.99 & 7.60 & 8.01 & 8.30 & 7.96 & 6.66 \\
\hline 9 & 8.01 & 8.80 & 8.18 & 9.06 & 9.29 & 7.85 & 6.90 & 7.34 & 6.94 & 7.63 & 7.86 & 6.46 \\
\hline $\mathrm{Y}_{\mathrm{ij}}$ & 6.40 & 6.57 & 7.45 & 7.15 & 7.10 & $10.75^{\tau}$ & 5.46 & 5.60 & 6.39 & 5.94 & 6.04 & $8.99^{\tau}$ \\
\hline
\end{tabular}

${ }^{\tau}$ Check: mean of 48 plots in four replications.

Genetics and Molecular Research 16 (1): gmr16019544 
C.M. Silva et al.

Table 2. Observed means of ten traits in a partial diallel between two sets of maize populations (2nd crop).

\begin{tabular}{|c|c|c|c|c|c|c|c|c|c|c|c|c|}
\hline $\mathrm{ilj}$ & $1^{\prime}$ & $2^{\prime}$ & $3^{\prime}$ & $4^{\prime}$ & $5^{\prime}$ & $\mathrm{Y}_{\mathrm{ii}}$ & 1' $^{\prime}$ & $2^{\prime}$ & $3^{\prime}$ & $4^{\prime}$ & $5^{\prime}$ & $\mathrm{Y}_{\mathrm{ii}}$ \\
\hline & \multicolumn{6}{|c|}{ MF - Male flowering (days) } & \multicolumn{6}{|c|}{ SD - Stalk diameter (mm) } \\
\hline 1 & 59.5 & 59.5 & 60.5 & 59.8 & 59.3 & 60.0 & 23.6 & 25.4 & 26.2 & 25.0 & 24.7 & 24.3 \\
\hline 2 & 59.5 & 59.0 & 60.3 & 61.0 & 61.3 & 59.5 & 25.0 & 24.3 & 24.7 & 25.1 & 25.5 & 23.1 \\
\hline 3 & 59.0 & 59.3 & 60.8 & 59.5 & 61.3 & 60.3 & 24.1 & 25.9 & 23.4 & 23.9 & 26.8 & 24.7 \\
\hline 4 & 59.5 & 59.5 & 59.5 & 60.8 & 61.5 & 61.5 & 25.3 & 25.6 & 24.3 & 24.4 & 25.9 & 25.8 \\
\hline 5 & 60.0 & 59.0 & 59.0 & 59.5 & 59.5 & 59.3 & 23.4 & 23.4 & 22.6 & 22.9 & 24.7 & 23.5 \\
\hline 6 & 59.0 & 60.3 & 62.0 & 60.5 & 60.5 & 60.5 & 25.8 & 24.7 & 25.3 & 24.9 & 26.8 & 26.3 \\
\hline 7 & 59.0 & 60.3 & 61.0 & 60.8 & 60.8 & 59.5 & 23.6 & 24.9 & 24.6 & 23.6 & 25.4 & 23.7 \\
\hline 8 & 59.8 & 59.8 & 60.3 & 59.3 & 60.8 & 61.5 & 25.4 & 24.0 & 24.8 & 24.3 & 26.1 & 23.8 \\
\hline 9 & 59.5 & 59.8 & 60.3 & 59.8 & 60.0 & 60.8 & 24.9 & 25.1 & 24.6 & 23.1 & 25.8 & 23.4 \\
\hline $\mathrm{Y}_{\mathrm{ij}}$ & 59.3 & 60.5 & 61.8 & 60.3 & 62.5 & $59.7^{\tau}$ & 23.1 & 25.0 & 22.5 & 25.0 & 24.5 & $24.5^{\tau}$ \\
\hline $\mathrm{ilj}$ & \multicolumn{6}{|c|}{ PH - Plant height (m) } & \multicolumn{6}{|c|}{ EH - Ear height (m) } \\
\hline 1 & 2.27 & 2.41 & 2.37 & 2.30 & 2.37 & 2.46 & 1.20 & 1.26 & 1.29 & 1.21 & 1.31 & 1.48 \\
\hline 2 & 2.24 & 2.29 & 2.32 & 2.22 & 2.40 & 2.40 & 1.14 & 1.28 & 1.33 & 1.24 & 1.41 & 1.39 \\
\hline 3 & 2.26 & 2.29 & 2.33 & 2.26 & 2.50 & 2.37 & 1.19 & 1.21 & 1.33 & 1.19 & 1.45 & 1.38 \\
\hline 4 & 2.18 & 2.29 & 2.38 & 2.32 & 2.42 & 2.51 & 1.15 & 1.27 & 1.32 & 1.37 & 1.38 & 1.45 \\
\hline 5 & 2.12 & 2.29 & 2.32 & 2.31 & 2.24 & 2.51 & 1.14 & 1.30 & 1.28 & 1.27 & 1.21 & 1.49 \\
\hline 6 & 2.29 & 2.49 & 2.51 & 2.34 & 2.56 & 2.65 & 1.28 & 1.51 & 1.50 & 1.40 & 1.52 & 1.63 \\
\hline 7 & 2.13 & 2.37 & 2.52 & 2.30 & 2.44 & 2.55 & 1.12 & 1.29 & 1.43 & 1.31 & 1.40 & 1.56 \\
\hline 8 & 2.45 & 2.33 & 2.42 & 2.47 & 2.50 & 2.63 & 1.38 & 1.31 & 1.40 & 1.53 & 1.51 & 1.58 \\
\hline 9 & 2.38 & 2.36 & 2.38 & 2.25 & 2.38 & 2.67 & 1.21 & 1.35 & 1.41 & 1.32 & 1.38 & 1.64 \\
\hline \multirow[t]{2}{*}{$\mathrm{Y}_{\mathrm{j} j}$} & 1.72 & 1.94 & 2.08 & 1.85 & 1.97 & $2.47^{\tau}$ & 0.84 & 1.02 & 1.20 & 0.99 & 0.98 & $1.27^{\tau}$ \\
\hline & \multicolumn{6}{|c|}{ TL - Tassel length $(\mathrm{cm})$} & \multicolumn{6}{|c|}{ NB - Tassel branch number } \\
\hline 1 & 36.8 & 38.3 & 37.2 & 38.3 & 38.0 & 39.9 & 12.3 & 12.5 & 13.0 & 13.1 & 11.9 & 16.3 \\
\hline 2 & 37.4 & 39.6 & 37.1 & 38.3 & 38.2 & 38.0 & 11.7 & 13.3 & 12.4 & 11.2 & 11.5 & 11.6 \\
\hline 3 & 39.6 & 38.5 & 37.6 & 38.3 & 39.1 & 40.2 & 11.3 & 13.5 & 12.7 & 12.1 & 13.2 & 12.1 \\
\hline 4 & 38.3 & 38.8 & 38.1 & 37.8 & 38.0 & 40.4 & 12.6 & 11.6 & 13.5 & 12.7 & 11.2 & 17.5 \\
\hline 5 & 35.8 & 38.3 & 37.6 & 38.2 & 36.1 & 34.7 & 10.8 & 11.3 & 13.8 & 14.3 & 14.0 & 16.1 \\
\hline 6 & 39.2 & 39.1 & 37.6 & 36.5 & 38.9 & 39.0 & 12.9 & 14.4 & 17.0 & 14.2 & 12.5 & 17.2 \\
\hline 7 & 40.7 & 39.9 & 39.6 & 38.4 & 40.3 & 38.3 & 12.3 & 13.4 & 12.2 & 11.1 & 10.3 & 19.2 \\
\hline 8 & 38.2 & 39.7 & 40.0 & 39.5 & 39.2 & 40.0 & 12.2 & 13.0 & 14.4 & 14.3 & 9.1 & 17.7 \\
\hline 9 & 36.8 & 37.7 & 35.9 & 37.8 & 38.3 & 36.1 & 11.4 & 14.4 & 14.6 & 12.0 & 10.7 & 17.0 \\
\hline \multirow[t]{2}{*}{$\mathrm{Y}_{\mathrm{j} j}$} & 33.91 & 32.49 & 34.95 & 35.70 & 37.53 & $39.2^{\tau}$ & 7.7 & 11.8 & 10.4 & 9.1 & 7.1 & $7.2^{\tau}$ \\
\hline & \multicolumn{6}{|c|}{ EL - Ear length $(\mathrm{cm})$} & \multicolumn{6}{|c|}{ ED - Ear diameter $(\mathrm{cm})$} \\
\hline 1 & 15.6 & 15.9 & 14.0 & 15.5 & 17.4 & 15.1 & 4.40 & 4.45 & 4.30 & 4.15 & 4.40 & 4.30 \\
\hline 2 & 14.9 & 15.5 & 15.4 & 14.5 & 16.1 & 15.8 & 4.25 & 4.20 & 4.35 & 4.10 & 4.50 & 4.20 \\
\hline 3 & 15.7 & 14.8 & 15.5 & 16.0 & 16.2 & 15.5 & 4.51 & 4.25 & 4.20 & 4.25 & 4.20 & 4.10 \\
\hline 4 & 16.6 & 14.7 & 16.0 & 15.3 & 17.3 & 14.4 & 4.25 & 4.30 & 4.20 & 4.15 & 4.35 & 4.20 \\
\hline 5 & 15.1 & 14.7 & 14.9 & 14.2 & 16.0 & 13.6 & 3.80 & 4.18 & 4.15 & 4.10 & 4.10 & 4.03 \\
\hline 6 & 16.5 & 15.4 & 16.3 & 16.0 & 15.8 & 15.6 & 4.05 & 4.20 & 4.45 & 4.30 & 4.45 & 4.20 \\
\hline 7 & 14.5 & 14.3 & 16.1 & 15.6 & 15.1 & 14.4 & 4.15 & 4.40 & 4.25 & 4.30 & 4.35 & 4.20 \\
\hline 8 & 15.9 & 14.8 & 16.1 & 15.0 & 15.6 & 15.3 & 4.20 & 4.15 & 4.15 & 4.40 & 4.30 & 3.95 \\
\hline 9 & 16.0 & 14.6 & 15.5 & 15.0 & 13.4 & 14.8 & 4.39 & 4.35 & 4.25 & 4.15 & 4.20 & 4.05 \\
\hline \multirow[t]{2}{*}{$Y_{j j}$} & 14.0 & 12.4 & 13.6 & 15.3 & 12.5 & $14.5^{\tau}$ & 4.00 & 4.30 & 4.20 & 4.13 & 3.80 & $4.45^{\tau}$ \\
\hline & \multicolumn{6}{|c|}{ EY - Ear yield (t/ha) } & \multicolumn{6}{|c|}{ GY - Grain yield (t/ha) } \\
\hline 1 & 6.46 & 6.35 & 6.52 & 6.26 & 6.91 & 6.16 & 5.55 & 5.29 & 5.48 & 5.22 & 5.93 & 5.22 \\
\hline 2 & 6.20 & 6.23 & 6.67 & 5.56 & 6.76 & 6.49 & 5.21 & 5.35 & 5.67 & 4.62 & 5.73 & 5.42 \\
\hline 3 & 6.17 & 6.63 & 6.21 & 5.81 & 6.34 & 6.00 & 5.09 & 5.58 & 5.26 & 4.84 & 5.26 & 5.01 \\
\hline 4 & 6.05 & 6.05 & 6.65 & 6.32 & 6.35 & 5.46 & 5.14 & 5.10 & 5.66 & 5.24 & 5.34 & 4.58 \\
\hline 5 & 5.37 & 5.68 & 6.10 & 5.41 & 5.63 & 5.15 & 4.46 & 4.84 & 5.13 & 4.43 & 4.70 & 4.22 \\
\hline 6 & 6.53 & 6.11 & 6.83 & 6.38 & 7.53 & 6.25 & 5.48 & 5.26 & 5.68 & 5.38 & 6.39 & 5.25 \\
\hline 7 & 6.12 & 5.76 & 7.12 & 6.77 & 6.56 & 5.12 & 5.09 & 4.82 & 6.05 & 5.62 & 5.65 & 4.27 \\
\hline 8 & 6.27 & 6.14 & 6.44 & 6.96 & 6.48 & 5.36 & 5.35 & 5.28 & 5.47 & 5.77 & 5.46 & 4.52 \\
\hline 9 & 6.11 & 5.77 & 6.32 & 5.77 & 5.74 & 5.37 & 5.18 & 4.88 & 5.40 & 4.72 & 4.90 & 4.43 \\
\hline $\mathrm{Y}_{\mathrm{j} j}$ & 5.02 & 5.26 & 6.09 & 5.44 & 5.08 & $7.58^{\tau}$ & 4.26 & 4.48 & 5.25 & 4.73 & 4.22 & $6.32^{\tau}$ \\
\hline
\end{tabular}

${ }^{\tau}$ Check: mean of 48 plots in four replications.

Genetics and Molecular Research 16 (1): gmr16019544 
Table 3. Observed means of ten traits in partial diallel cross $(9 \times 5)$ in two planting seasons $\left(\varepsilon_{1}, \varepsilon_{2}\right)^{1}$.

\begin{tabular}{l|c|c|c|c|c|c|c|c}
\hline & \multicolumn{2}{|c|}{ Varieties I } & \multicolumn{2}{c|}{ Varieties II } & \multicolumn{2}{c|}{ Hybrids } & \multicolumn{2}{c}{ Diallel $^{2}$} \\
\hline & $\varepsilon_{1}$ & $\varepsilon_{2}$ & $\varepsilon_{1}$ & $\varepsilon_{2}$ & $\varepsilon_{1}$ & $\varepsilon_{2}$ & $\varepsilon_{1}$ & $\varepsilon_{2}$ \\
\hline MF & 58.97 & 60.31 & 58.90 & 60.85 & 59.01 & 60.00 & 59.00 & 60.12 \\
\hline SD & 25.48 & 24.29 & 25.55 & 24.00 & 25.75 & 24.74 & 25.70 & 24.61 \\
\hline PH & 2.527 & 2.527 & 1.880 & 1.911 & 2.329 & 2.346 & 2.321 & 2.337 \\
\hline EH & 1.554 & 1.511 & 1.000 & 1.004 & 1.348 & 1.316 & 1.350 & 1.320 \\
\hline TL & 39.40 & 38.51 & 36.46 & 34.91 & 38.80 & 38.28 & 38.70 & 38.03 \\
\hline NB & 17.24 & 16.06 & 10.68 & 9.21 & 14.09 & 12.60 & 14.28 & 12.84 \\
\hline EL & 16.97 & 14.93 & 15.32 & 13.55 & 16.87 & 15.43 & 16.75 & 15.19 \\
\hline ED & 4.550 & 4.136 & 4.553 & 4.085 & 4.714 & 4.256 & 4.676 & 4.223 \\
\hline EY & 8.140 & 5.706 & 6.932 & 5.376 & 8.973 & 6.275 & 8.673 & 6.112 \\
\hline GY & 6.821 & 4.769 & 5.884 & 4.589 & 7.610 & 5.287 & 7344 & 5.149 \\
\hline
\end{tabular}

${ }^{1}$ Seasons: $\varepsilon_{1}(1$ st crop $=$ safra $)$ and $\varepsilon_{2}(2$ nd crop $=$ safrinha $) .{ }^{2}$ Diallel: refers to the whole set (Varieties I, Varieties II and Hybrids). For abbreviations, see Tables 1 or 2.

The relatively lower expression of EL and ED in Group II is explained because this group was developed from recombination of commercial hybrids and this procedure leads to a decrease in the level of heterozygosity and consequently in the hybrid vigor $(6.40 \%$ for EL and $3.56 \%$ for ED, on the average of two seasons; see Table 4). Comparing the sets (Group I, Group II, Hybrids), the highest means (cm) for EL were $(18.0,17.5,18.4)$ in $\varepsilon_{1}$ and $(15.8$, $15.3,17.4)$ in $\varepsilon_{2}$; for $\operatorname{ED}$ were $(4.75,4.67,4.95)$ in $\varepsilon_{1}$ and $(4.30,4.30,4.51)$ in $\varepsilon_{2}$. In the group of hybrids, the highest means for EL were in the crosses 9 x 5' (NAP-CS x HG-81]) and $1 \mathrm{x}$ 5' (NAP-FA x HG-81); and for ED were in the crosses 6 x 5' (NAP-PP x HG-81) and $3 \times 1$ ' (NAP-FB x HG-40).

Table 4. Average heterosis $(\overline{\mathrm{h}})$ for ten traits in a partial diallel cross between two groups of varieties evaluated in two planting seasons $\left(\varepsilon_{1}, \varepsilon_{2}\right)$.

\begin{tabular}{|c|c|c|c|c|c|c|c|c|c|}
\hline \multirow[t]{2}{*}{ Traits } & \multicolumn{2}{|c|}{ Season $\varepsilon_{1}$} & \multicolumn{2}{|c|}{ Season $\varepsilon_{2}$} & \multirow[t]{2}{*}{ Traits } & \multicolumn{2}{|c|}{ Season $\varepsilon_{1}$} & \multicolumn{2}{|c|}{ Season $\varepsilon_{2}$} \\
\hline & $\overline{\mathrm{h}}$ & $\overline{\mathrm{h}} \%$ & $\overline{\mathrm{h}}$ & $\overline{\mathrm{h}} \%$ & & $\overline{\mathrm{h}}$ & $\overline{\mathrm{h}} \%$ & $\overline{\mathrm{h}}$ & $\overline{\mathrm{h}} \%$ \\
\hline MF & 0.075 & 0.13 & -0.578 & -0.95 & NB & 0.131 & 0.94 & -0.036 & -0.29 \\
\hline SD & 0.241 & 0.94 & 0.594 & 2.46 & $\overline{E L}$ & 0.719 & 4.46 & 1.190 & 8.36 \\
\hline $\mathrm{PH}$ & 0.126 & 5.72 & 0.127 & 5.70 & ED & 0.163 & 3.58 & 0.146 & 3.54 \\
\hline EH & 0.071 & 5.57 & 0.059 & 4.70 & EY & 1.437 & 19.06 & 0.734 & 13.25 \\
\hline $\mathrm{TL}$ & 0.877 & 2.31 & 1.566 & 4.26 & GY & 1.262 & 19.80 & 0.609 & 13.00 \\
\hline
\end{tabular}

For abbreviations, see Tables 1 or 2 . Seasons: $\mathrm{e}_{1}-1$ st crop $=$ safra, $\mathrm{e}_{2}-2$ nd crop $=$ safrinha.

In season $\mathrm{e}_{1}$, the highest yielding varieties (ear yield and grain yield) were 1 (NAP-FA) and 4 (NAP-DB) in Group I, 3' (HG-55) in Group II and the hybrid 7 x 3' (NAP-PZ x HG-55). In season $\mathrm{e}_{2}$, the outstanding materials were 2 (NAP-FL), 3' (HG-55) and 6 x 3' (NAP-PP x HG-55), respectively. On average, Group I showed ear yield 17.4 and $6.1 \%$ higher than Group II in $\mathrm{e}_{1}$ and $\mathrm{e}_{2}$, respectively; for grain yield the corresponding values were 15.9 and $3.9 \%$.

The average heterosis $(\overline{\mathrm{h}})$ expressed in variety crosses were below $5 \%$ (relative to midparent) in most instances (Table 4); for plant traits $\overline{\mathrm{h}}>5 \%$ were detected only for PH in both seasons, $\mathrm{EH}$ in $\mathrm{e}_{1}$ and $\mathrm{EL}$ in $\mathrm{e}_{2}$. The yield traits (EY and GY) showed midparent heterosis around $19 \%$ in $\mathrm{e}_{1}$ and around $13 \%$ in $\mathrm{e}_{2}$. As already mentioned, estimates of heterosis of GY for specific crosses were used for prediction of the population resulting from recombination of $\mathrm{F}_{1}$ hybrids between varieties (NAP x HG). Therefore, estimates of heterosis for GY are shown in Table 5, for all crosses. 
Table 5. Heterosis $\left(\mathrm{h}_{\mathrm{ij}}\right)$ for grain yield $(\mathrm{t} / \mathrm{ha})$ of hybrids $\left(\mathrm{H}_{\mathrm{ij}}\right)$ in a partial diallel evaluated in two planting seasons.

\begin{tabular}{|c|c|c|c|c|c|c|c|c|c|c|c|}
\hline $\mathrm{H}_{\mathrm{ij}}$ & $\mathrm{h}_{\mathrm{ij}}$ & $\mathrm{h}_{\mathrm{ijj}} \%$ & $\mathrm{H}_{\mathrm{ij}}$ & $\mathrm{h}_{\mathrm{ij}}$ & $\mathrm{h}_{\mathrm{ijj}} \%$ & $\mathrm{H}_{\mathrm{ij}}$ & $\mathrm{h}_{\mathrm{ij}}$ & $\mathrm{h}_{\mathrm{ijj}} \%$ & $\mathrm{H}_{\mathrm{ij}}$ & $\mathrm{h}_{\mathrm{ij}}$ & $\mathrm{h}_{\mathrm{ijj}} \%$ \\
\hline \multicolumn{12}{|c|}{ Season $\varepsilon_{1}$} \\
\hline $\mathrm{H}_{11^{\prime}}$ & 0.539 & 8.4 & $\mathrm{H}_{33^{\prime}}$ & 1.468 & 22.5 & $\mathrm{H}_{55^{\prime}}$ & 1.581 & 26.4 & $\mathrm{H}_{82^{\prime}}$ & 1.470 & 24.0 \\
\hline $\mathrm{H}_{12^{\prime}}$ & 0.969 & 15.0 & $\mathrm{H}_{34^{\prime}}$ & 1.302 & 20.7 & $\mathrm{H}_{61^{\prime}}$ & 1.691 & 26.9 & $\mathrm{H}_{83^{\prime}}$ & 1.482 & 22.7 \\
\hline $\mathrm{H}_{13^{\prime}}$ & 0.110 & 1.6 & $\mathrm{H}_{35^{\prime}}$ & 2.067 & 32.5 & $\mathrm{H}_{62^{\prime}}$ & 1.298 & 20.4 & $\mathrm{H}_{84^{\prime}}$ & 2.006 & 31.9 \\
\hline $\mathrm{H}_{14^{\prime}}$ & 1.256 & 18.9 & $\mathrm{H}_{41^{\prime}}$ & 1.198 & 18.6 & $\mathrm{H}_{63^{\prime}}$ & 0.976 & 14.5 & $\mathrm{H}_{85^{\prime}}$ & 1.616 & 25.5 \\
\hline $\mathrm{H}_{15^{\prime}}$ & 1.785 & 26.7 & $\mathrm{H}_{42}$ & 1.432 & 22.0 & $\mathrm{H}_{64^{\prime}}$ & 1.406 & 21.5 & $\mathrm{H}_{91^{\prime}}$ & 0.942 & 15.8 \\
\hline $\mathrm{H}_{21^{\prime}}$ & 1.768 & 28.1 & $\mathrm{H}_{43}$ & -0.251 & -3.6 & $\mathrm{H}_{65^{\prime}}$ & 1.853 & 28.2 & $\mathrm{H}_{92}$ & 1.312 & 21.8 \\
\hline $\mathrm{H}_{22}$ & 1.302 & 20.5 & $\mathrm{H}_{44}$ & 1.322 & 19.8 & $\mathrm{H}_{71^{\prime}}$ & 1.017 & 16.8 & $\mathrm{H}_{93^{\prime}}$ & 0.515 & 8.0 \\
\hline $\mathrm{H}_{23^{\prime}}$ & 0.208 & 3.1 & $\mathrm{H}_{45^{\prime}}$ & 0.978 & 14.5 & $\mathrm{H}_{72^{\prime}}$ & 0.975 & 15.9 & $\mathrm{H}_{94}$ & 1.426 & 23.0 \\
\hline $\mathrm{H}_{24^{\prime}}$ & 0.637 & 9.8 & $\mathrm{H}_{51^{\prime}}$ & 0.874 & 15.3 & $\mathrm{H}_{73^{\prime}}$ & 2.348 & 36.0 & $\mathrm{H}_{95}$ & 1.610 & 25.8 \\
\hline $\mathrm{H}_{25^{\prime}}$ & 1.620 & 24.6 & $\mathrm{H}_{52}$ & 1.016 & 17.6 & $\mathrm{H}_{74^{\prime}}$ & 1.636 & 26.0 & Mean & 1.258 & 19.8 \\
\hline $\mathrm{H}_{31^{\prime}}$ & 1.332 & 22.0 & $\mathrm{H}_{53}$ & 1.383 & 22.4 & $\mathrm{H}_{75^{\prime}}$ & 1.049 & 16.5 & $\operatorname{Max}$ & 2.348 & 36.0 \\
\hline $\mathrm{H}_{32}$ & 1.174 & 19.1 & $\mathrm{H}_{54^{\prime}}$ & 0.974 & 16.4 & $\mathrm{H}_{81}$ & 1.936 & 32.0 & Min & -0.251 & -3.6 \\
\hline \multicolumn{12}{|c|}{ Season $\varepsilon_{2}$} \\
\hline $\mathrm{H}_{11^{\prime}}$ & 0.803 & 16.9 & $\mathrm{H}_{33^{\prime}}$ & 0.122 & 2.4 & $\mathrm{H}_{55^{\prime}}$ & 0.479 & 11.4 & $\mathrm{H}_{82}$ & 0.774 & 17.2 \\
\hline $\mathrm{H}_{12^{\prime}}$ & 0.437 & 9.0 & $\mathrm{H}_{34}$ & -0.038 & -0.8 & $\mathrm{H}_{61^{\prime}}$ & 0.720 & 15.1 & $\mathrm{H}_{83^{\prime}}$ & 0.584 & 11.9 \\
\hline $\mathrm{H}_{13^{\prime}}$ & 0.245 & 4.7 & $\mathrm{H}_{35^{\prime}}$ & 0.646 & 14.0 & $\mathrm{H}_{62^{\prime}}$ & 0.390 & 8.0 & $\mathrm{H}_{84^{\prime}}$ & 1.139 & 24.6 \\
\hline $\mathrm{H}_{14}$ & 0.248 & 5.0 & $\mathrm{H}_{41^{\prime}}$ & 0.723 & 16.4 & $\mathrm{H}_{63^{\prime}}$ & 0.428 & 8.2 & $\mathrm{H}_{85^{\prime}}$ & 1.085 & 24.8 \\
\hline $\mathrm{H}_{15^{\prime}}$ & 1.212 & 25.7 & $\mathrm{H}_{42^{\prime}}$ & 0.566 & 12.5 & $\mathrm{H}_{64^{\prime}}$ & 0.391 & 7.8 & $\mathrm{H}_{91^{\prime}}$ & 0.831 & 19.2 \\
\hline $\mathrm{H}_{21^{\prime}}$ & 0.372 & 7.7 & $\mathrm{H}_{43^{\prime}}$ & 0.748 & 15.2 & $\mathrm{H}_{65^{\prime}}$ & 1.658 & 35.0 & $\mathrm{H}_{92^{\prime}}$ & 0.427 & 9.6 \\
\hline $\mathrm{H}_{22^{\prime}}$ & 0.401 & 8.1 & $\mathrm{H}_{44^{\prime}}$ & 0.588 & 12.6 & $\mathrm{H}_{71^{\prime}}$ & 0.826 & 19.4 & $\mathrm{H}_{93^{\prime}}$ & 0.555 & 11.5 \\
\hline $\mathrm{H}_{23^{\prime}}$ & 0.335 & 6.3 & $\mathrm{H}_{45}$ & 0.939 & 21.4 & $\mathrm{H}_{72^{\prime}}$ & 0.443 & 10.1 & $\mathrm{H}_{94}$ & 0.141 & 3.1 \\
\hline $\mathrm{H}_{24^{\prime}}$ & -0.456 & -9.0 & $\mathrm{H}_{51^{\prime}}$ & 0.218 & 5.2 & $\mathrm{H}_{73^{\prime}}$ & 1.287 & 27.0 & $\mathrm{H}_{95}$ & 0.575 & 13.3 \\
\hline $\mathrm{H}_{25^{\prime}}$ & 0.913 & 18.9 & $\mathrm{H}_{52^{\prime}}$ & 0.486 & 11.2 & $\mathrm{H}_{74^{\prime}}$ & 1.122 & 24.9 & Mean & 0.609 & 13.0 \\
\hline $\mathrm{H}_{31^{\prime}}$ & 0.456 & 9.8 & $\mathrm{H}_{53}$ & 0.400 & 8.5 & $\mathrm{H}_{75^{\prime}}$ & 1.407 & 33.2 & Max & 1.658 & 35.0 \\
\hline $\mathrm{H}_{32^{\prime}}$ & 0.835 & 17.6 & $\mathrm{H}_{54}$ & -0.045 & -1.0 & $\mathrm{H}_{81^{\prime}}$ & 0.960 & 21.9 & Min & -0.456 & -9.0 \\
\hline
\end{tabular}

The preliminary analysis of variance referring only to treatments of the diallel scheme is shown in Table 6 for the ten analyzed traits. The effects of replications were significant for seven traits in season $\mathrm{e}_{1}$ and for three traits in $\mathrm{e}_{2}$. The mean squares for populations were nonsignificant only for SD (stalk diameter) in season $e_{1}$. The mean squares of the error term were adjusted to be used in the analysis of treatment means (over four replications) according to the partial diallel model shown in Tables 7 and 8 for season $\mathrm{e}_{1}$. The variation among hybrids was significant for all traits except for SD. The effects of GCA of varieties in Group I were nonsignificant for SD, TL, EL and ED; in Group II the GCA effects were significant for all traits. The effects of SCA were significant only for MF, PH, EH, EY, and GY. The differences among varieties of Group I were non-significant only for SD, TL, EL and GY, while in Group II nonsignificants were for SD, TL, ED, and GY. The contrast (v) for comparing the two groups of varieties were non-significant for MF, SD, TL and $\mathrm{ED}$. The contrast (h) for comparing hybrids $v s$ varieties is related to the average heterosis of the hybrid crosses and were non-significant only for MF, SD, PH, EH and TL. In season $\mathrm{e}_{2}$ (Tables 9 and 10), the GCA effects were nonsignificant only for NB in Group I and for TL and ED in Group II. The SCA effects were nonsignificant for SD, TL and NB. Non-significance also was found for SD, ED and GY in the contrast (v); and for EH in the contrast (h). The level of significant differentiation expressed in the groups of varieties and variety crosses, mainly in relation to the effects of combining ability and heterosis, may assure an acceptable confidence to use the methodology for prediction of the performance of the new populations derived from the source of germplasm under study. The use of prediction methods for the synthesis of hybrids from inbred lines (Jenkins, 1934, 1978) and composite populations of crosses between composites (Eberhart et al., 1967; Miranda Filho and Chaves, 1991) have been used in maize-breeding programs.

Genetics and Molecular Research 16 (1): gmr16019544 
Table 6. Preliminary analysis of variance of ten traits in a partial diallel between two groups of varieties in two crop seasons.

\begin{tabular}{l|c|c|c|c|c|c|c|c|c|c}
\hline & \multicolumn{9}{|c}{ 1st crop (safra) } & \multicolumn{4}{c}{ 2nd crop (safrinha) } \\
\hline Trait & Reps. $^{\text {a }}$ & Pops. $^{\text {a }}$ & Error $^{\mathrm{a}}$ & Mean & CV\% $^{\ddagger}$ & Reps. $^{\mathrm{a}}$ & Pops. $^{\mathrm{a}}$ & Error $^{\mathrm{a}}$ & Mean $^{\ddagger}$ & CV\% $^{\ddagger}$ \\
\hline MF & $7.959^{\ddagger}$ & $4.237^{\ddagger}$ & 1.238 & 59.0 & 1.89 & $1.209^{\eta}$ & $2.865^{\ddagger}$ & 0.798 & 60.1 & 1.49 \\
\hline SD & $3.054^{\eta}$ & $3.306^{\eta}$ & 2.834 & 25.7 & 6.55 & $40.00^{\ddagger}$ & $4.272^{\ddagger}$ & 2.004 & 24.6 & 5.75 \\
\hline PH & $4.920^{+}$ & $13.66^{\ddagger}$ & 1.383 & 2.32 & 5.07 & $2.926^{+}$ & $12.74^{\ddagger}$ & 0.976 & 2.34 & 4.23 \\
\hline EH & $3.692^{+}$ & $11.52^{\ddagger}$ & 1.098 & 1.35 & 7.76 & $0.730^{\eta}$ & $10.35^{\ddagger}$ & 0.760 & 1.32 & 6.61 \\
\hline TL & $20.70^{\ddagger}$ & $8.191^{\ddagger}$ & 4.364 & 38.7 & 5.40 & $1.956^{\eta}$ & $10.83^{\ddagger}$ & 4.822 & 38.0 & 5.77 \\
\hline NB & $4.616^{\eta}$ & $19.76^{\ddagger}$ & 3.883 & 14.3 & 13.8 & $1.661^{\eta}$ & $21.89^{\ddagger}$ & 5.829 & 12.8 & 18.8 \\
\hline EL & $0.967^{\eta}$ & $3.055^{\ddagger}$ & 1,247 & 16.8 & 6.67 & $2.395^{\eta}$ & $3.864^{\ddagger}$ & 1.113 & 15.2 & 6.94 \\
\hline ED & $17.40^{\ddagger}$ & $9.078^{\ddagger}$ & 3.279 & 4.68 & 3.87 & $32.23^{\ddagger}$ & $8.794^{\ddagger}$ & 3.839 & 4.22 & 4.64 \\
\hline EY & $136.6^{\ddagger}$ & $58.77^{\ddagger}$ & 10.46 & 3.90 & 8.29 & $8.689^{\eta}$ & $24.24^{\ddagger}$ & 5.588 & 2.75 & 8.59 \\
\hline GY & $145.8^{\ddagger}$ & $43.42^{\ddagger}$ & 9.266 & 3.30 & 9.21 & $3.814^{\eta}$ & $18.77^{\ddagger}$ & 4.339 & 2.32 & 8.99 \\
\hline
\end{tabular}

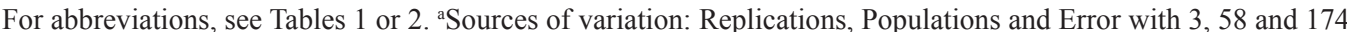
degrees of freedom. PH, EH, ED, EY, and GY: mean squares multiplied by $10^{2} . \mathrm{CV}=$ coefficient of variation.

Table 7. Mean squares ${ }^{\mathrm{a}}$ in the analysis of variance $^{\mathrm{b}}$ of five traits in a partial diallel between two groups of varieties (1st crop).

\begin{tabular}{l|c|c|c|c|c|c}
\hline Source & d.f. & MF & SD & PH & EH & TL \\
\hline Populations & 58 & $105.9^{\ddagger}$ & $82.64^{\eta}$ & $34.15^{\ddagger}$ & $28.81^{\ddagger}$ & $20.48^{\ddagger}$ \\
\hline Hybrids (H) & 44 & $110.8^{\ddagger}$ & $95.18^{\eta}$ & $11.83^{\ddagger}$ & $12.85^{\ddagger}$ & $17.67^{\ddagger}$ \\
\hline GCA-I & 8 & $165.9^{\ddagger}$ & $48.50^{\eta}$ & $24.39^{\ddagger}$ & $27.12^{\ddagger}$ & $18.11^{\eta}$ \\
\hline GCA-II & 4 & $443.6^{\ddagger}$ & $361.7^{\ddagger}$ & $33.45^{\ddagger}$ & $38.40^{\ddagger}$ & $39.78^{\ddagger}$ \\
\hline SCA & 32 & $55.41^{\ddagger}$ & $73.54^{\eta}$ & $5.990^{+}$ & $6.088^{\ddagger}$ & $14.80^{\eta}$ \\
\hline Varieties I & 8 & $64.76^{+}$ & $58.87^{\eta}$ & $7.550^{+}$ & $11.75^{\ddagger}$ & $9.324^{\eta}$ \\
\hline Varieties II & 4 & $186.3^{\ddagger}$ & $16.53^{\eta}$ & $9.638^{+}$ & $6.704^{+}$ & $8.759^{\eta}$ \\
\hline Groups & 2 & $3.072^{\eta}$ & $34.02^{\eta}$ & $680.5^{\ddagger}$ & $492.5^{\ddagger}$ & $150.2^{\ddagger}$ \\
\hline Contrast (v) & 1 & $1.677^{\eta}$ & $1.373^{\eta}$ & $1349^{\ddagger}$ & $984.3^{\ddagger}$ & $278.2^{\ddagger}$ \\
\hline Contrast (h) & 1 & $4.467^{\eta}$ & $66.68^{\eta}$ & $11.92^{\eta}$ & $0.676^{\eta}$ & $22.27^{\eta}$ \\
\hline Error & 174 & 30.94 & 70.86 & 3.458 & 2.746 & 10.91 \\
\hline
\end{tabular}

${ }^{a}$ Mean squares multiplied by $10^{2}$ in MF, SD and TL; and by $10^{3}$ in PH and EH. ${ }^{b}$ Analysis with means of four replications in randomized blocks. For abbreviations, see Tables 1 or $2{ }^{{ }^{*},+, h}$ Significance by F-test: $\mathrm{P}<0.01, \mathrm{P}<0.05$ and no significance, respectively. Contrasts: $\vee \bar{Y}_{I}-\bar{Y}_{I I}=; \mathrm{h}=\bar{Y}_{H}-\bar{Y}_{V}$ (See Material and Methods).

Table 8. Mean squares ${ }^{\mathrm{a}}$ in the analysis of variance ${ }^{\mathrm{b}}$ of five traits in a partial diallel between two groups of varieties (1st crop).

\begin{tabular}{l|c|c|c|c|c|c}
\hline Source & d.f. & NB & EL & ED & EY & GY \\
\hline Populations & 58 & $49.40^{\ddagger}$ & $7.637^{\ddagger}$ & $22.69^{\ddagger}$ & $14.69^{\ddagger}$ & $10.86^{\ddagger}$ \\
\hline Hybrids (H) & 44 & $24.05^{\ddagger}$ & $5.136^{\ddagger}$ & $15.64^{\ddagger}$ & $7.939^{\ddagger}$ & $5.724^{\ddagger}$ \\
\hline GCA-I & 8 & $31.87^{\ddagger}$ & $2.566^{\eta}$ & $16.01^{\eta}$ & $11.05^{\ddagger}$ & $7.903^{\ddagger}$ \\
\hline GCA-II & 4 & $106.3^{\ddagger}$ & $17.24^{\ddagger}$ & $54.81^{\ddagger}$ & $17.23^{\ddagger}$ & $11.52^{\ddagger}$ \\
\hline SCA & 32 & $11.81^{\eta}$ & $4.265^{\eta}$ & $10.65^{\eta}$ & $5.999^{\ddagger}$ & $4.455^{\ddagger}$ \\
\hline Varieties I & 8 & $30.79^{\ddagger}$ & $3.226^{\eta}$ & $39.37^{\ddagger}$ & $5.866^{+}$ & $4.521^{\eta}$ \\
\hline Varieties II & 4 & $27.02^{+}$ & $19.61^{\ddagger}$ & $7.139^{\eta}$ & $3.849^{\eta}$ & $2.805^{\eta}$ \\
\hline Groups & 2 & $726.3^{\ddagger}$ & $56.35^{\ddagger}$ & $142.3^{\ddagger}$ & $220.3^{\ddagger}$ & $165.2^{\ddagger}$ \\
\hline Contrast (v) & 1 & $1383^{\ddagger}$ & $87.74^{\ddagger}$ & $0.036^{\eta}$ & $94.99^{\ddagger}$ & $57.05^{\ddagger}$ \\
\hline Contrast (h) & 1 & $69.41^{\ddagger}$ & $24.95^{\ddagger}$ & $284.6^{\ddagger}$ & $345.5^{\ddagger}$ & $273.3^{\ddagger}$ \\
\hline Error & 174 & 9.707 & 3.117 & 8.196 & 2.616 & 2.316 \\
\hline
\end{tabular}

${ }^{\mathrm{a}}$ Mean squares multiplied by 10 in NB and EL; by $10^{2}$ in EY and GY; and by $10^{3}$ in ED. ${ }^{\mathrm{b} A n a l y s i s}$ with means of four replications in randomized blocks. For abbreviations, see Tables 1 or $2{ }^{{ }^{*}+, \text { h }}$ Significance by F-test: $\mathrm{P}<0.01, \mathrm{P}<$ 0.05 and no significance, respectively. Contrasts: $\mathrm{v}=\bar{Y}_{I}-\bar{Y}_{I I} ; \mathrm{h}=\bar{Y}_{H}-\bar{Y}_{V}$ (See Material and Methods).

Genetics and Molecular Research 16 (1): gmr16019544 
Table 9. Mean squares ${ }^{\mathrm{a}}$ in the analysis of variance $^{\mathrm{b}}$ of five traits in a partial diallel between two groups of varieties (2nd crop).

\begin{tabular}{l|c|c|c|c|c|c}
\hline Source & d.f. & MF & SD & PH & EH & TL \\
\hline Populations & 58 & $71.63^{\ddagger}$ & $106.8^{\ddagger}$ & $31.86^{\ddagger}$ & $25.88^{\ddagger}$ & $27.08^{\ddagger}$ \\
\hline Hybrids (H) & 44 & $58.52^{\ddagger}$ & $98.11^{\ddagger}$ & $10.14^{\ddagger}$ & $12.04^{\ddagger}$ & $13.21^{\eta}$ \\
\hline GCA-I & 8 & $54.06^{\ddagger}$ & $164.7^{\ddagger}$ & $17.93^{\ddagger}$ & $25.74^{\ddagger}$ & $37.19^{\ddagger}$ \\
\hline GCA-II & 4 & $213.9^{\ddagger}$ & $328.4^{\ddagger}$ & $39.34^{\ddagger}$ & $51.77^{\ddagger}$ & $13.76^{\eta}$ \\
\hline SCA & 32 & $40.22^{\ddagger}$ & $52.66^{\eta}$ & $4.547^{\ddagger}$ & $3.642^{\ddagger}$ & $7.146^{\eta}$ \\
\hline Varieties I & 8 & $69.97^{\ddagger}$ & $125.2^{+}$ & $11.51^{\ddagger}$ & $9.496^{\ddagger}$ & $39.11^{\ddagger}$ \\
\hline Varieties II & 4 & $164.4^{\ddagger}$ & $130.6^{+}$ & $18.26^{\ddagger}$ & $16.52^{\ddagger}$ & $35.80^{+}$ \\
\hline Groups & 2 & $181.1^{\ddagger}$ & $176.5^{+}$ & $618.2^{\ddagger}$ & $414.7^{\ddagger}$ & $266.5^{\ddagger}$ \\
\hline Contrast (v) & 1 & $95.28^{+}$ & $27.76^{\eta}$ & $1221^{\ddagger}$ & $827.6^{\ddagger}$ & $414.8^{\ddagger}$ \\
\hline Contrast (h) & 1 & $266.9^{\ddagger}$ & $325.2^{+}$ & $15.84^{+}$ & $1.920^{\eta}$ & $118.3^{\ddagger}$ \\
\hline Error & 174 & 19.95 & 50.10 & 2.441 & 1.900 & 12.05 \\
\hline
\end{tabular}

${ }^{a}$ Mean squares multiplied by $10^{2}$ in MF, SD and TL; and by $10^{3}$ in PH and EH. ${ }^{b}$ Analysis with means of four replications in randomized blocks. For abbreviations, see Tables 1 or $2{ }^{{ }^{*},+ \text {,h }}$ Significance by F-test: $\mathrm{P}<0.01, \mathrm{P}<0.05$ and no significance, respectively. Contrasts: $\mathrm{v}=\bar{Y}_{I}-\bar{Y}_{I I} ; \mathrm{h}=\bar{Y}_{H}-\bar{Y}_{V}$ (See Material and Methods).

Table 10. Mean squares ${ }^{\mathrm{a}}$ in the analysis of variance ${ }^{\mathrm{b}}$ of five traits in a partial diallel between two groups of varieties (2nd crop).

\begin{tabular}{l|c|c|c|c|c|c}
\hline Source & d.f. & NB & EL & ED & EY & GY \\
\hline Populations & 58 & $54.73^{\ddagger}$ & $9.660^{\ddagger}$ & $21.99^{\ddagger}$ & $6.061^{\ddagger}$ & $4.692^{\ddagger}$ \\
\hline Hybrids (H) & 44 & $20.14^{\eta}$ & $6.728^{\ddagger}$ & $18.51^{\ddagger}$ & $4.179^{\ddagger}$ & $3.364^{\ddagger}$ \\
\hline GCA-I & 8 & $22.60^{\eta}$ & $8.471^{\ddagger}$ & $29.72^{\ddagger}$ & $10.13^{\ddagger}$ & $8.031^{\ddagger}$ \\
\hline GCA-II & 4 & $66.64^{\ddagger}$ & $11.41^{\ddagger}$ & $16.19^{\eta}$ & $8.461^{\ddagger}$ & $7.644^{\ddagger}$ \\
\hline SCA & 32 & $13.71^{\eta}$ & $5.706^{\ddagger}$ & $15.99^{\ddagger}$ & $2.156^{+}$ & $1.662^{+}$ \\
\hline Varieties I & 8 & $65.20^{\ddagger}$ & $5.009^{\eta}$ & $12.36^{\eta}$ & $5.431^{\ddagger}$ & $4.267^{\ddagger}$ \\
\hline Varieties II & 4 & $37.71^{+}$ & $14.50^{\ddagger}$ & $37.37^{\ddagger}$ & $3.763^{+}$ & $3.620^{+}$ \\
\hline Groups & 2 & $808.1^{\ddagger}$ & $83.09^{\ddagger}$ & $106.2^{\ddagger}$ & $54.58^{\ddagger}$ & $37.77^{\ddagger}$ \\
\hline Contrast (v) & 1 & $1506^{\ddagger}$ & $60.77^{\ddagger}$ & $8.397^{\eta}$ & $7.137^{+}$ & $2.105^{\eta}$ \\
\hline Contrast (h) & 1 & $109.9^{\ddagger}$ & $105.4^{\ddagger}$ & $204.1^{\ddagger}$ & $102.0^{\ddagger}$ & $73.42^{\ddagger}$ \\
\hline Error & 174 & 14.57 & 2.783 & 9.597 & 1.397 & 1.085 \\
\hline
\end{tabular}

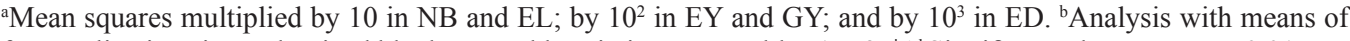
four replications in randomized blocks. For abbreviations, see Tables 1 or $2 .{ }^{\star,+h}$ Significance by F-test: $\mathrm{P}<0.01, \mathrm{P}<$ 0.05 and no significance, respectively. Contrasts: $\mathrm{v}=\bar{Y}_{I}-\bar{Y}_{I I} ; \mathrm{h}=\bar{Y}_{H}-\bar{Y}_{V}$ (See Material and Methods).

The estimates of the effects of GCA for both groups of varieties $\left(g_{i}-\right.$ group I; $g_{j}$ group II) and the maximum and minimum values for the estimates of SCA $\left(\mathrm{s}_{\mathrm{ij}}\right)$ within the group of 45 variety crosses are shown in Table 11. High estimates for GCA, either positive or negative, indicate more or less expression of the trait on the average of all crosses involving the respective parent; these effects are then related to the predominantly additive gene action (Sprague and Tatum, 1942). On the other hand, the $\mathrm{s}_{\mathrm{ij}}$ effect expresses the non-additive gene action and is a component of the observed mean in a hybrid combination.

As already emphasized by Vencovsky and Barriga (1992), the GCA effects are associated with the allele frequency in the parents, so that the higher frequencies of favorable alleles will lead to the positive expression of the trait and lower frequencies to the negative expression. Therefore, the breeder must be interested in positive expression (positive GCA) for some traits and negative expression (negative GCA) for other traits. For example, when focusing the agronomic aspect in maize, for traits such as male flowering, plant and ear height, tassel size, etc., should be desirable a decrease in their phenotypic expression; on the other hand, for traits such as ear length and diameter, grain yield, disease resistance, etc., a positive expression is desirable in most instances.

Genetics and Molecular Research 16 (1): gmr16019544 
Table 11. Estimates of the mean $\left(m_{H}\right)$, effects of general combining ability $\left(g_{i}, g_{j}\right)$, and range for specific combining ability $\left(\mathrm{A} / \mathrm{s}_{\mathrm{ij}}\right)$ for ten traits in hybrid crosses evaluated in two crop seasons.

\begin{tabular}{|c|c|c|c|c|c|c|c|c|c|c|}
\hline $\mathrm{g}_{\mathrm{i}, \mathrm{g}_{\mathrm{j}}}$ & \multicolumn{10}{|c|}{ 1st crop (safra) } \\
\hline$\overline{i, j}$ & MF & SD & $\mathrm{PH}$ & $\mathrm{EH}$ & TL & NB & $\overline{E L}$ & ED & EY & GY \\
\hline 1 & -0.26 & 0.18 & -0.08 & -0.10 & -0.20 & -1.19 & 0.39 & 0.08 & 0.047 & -0.065 \\
\hline 2 & 0.14 & -0.16 & -0.02 & 0.00 & -0.41 & -1.15 & -0.18 & 0.06 & -0.010 & 0.001 \\
\hline 3 & -0.06 & -0.20 & -0.03 & -0.03 & 0.61 & -0.09 & -0.22 & -0.05 & 0.162 & 0.141 \\
\hline 4 & -0.16 & 0.11 & -0.01 & -0.01 & 0.26 & -0.41 & -0.07 & -0.04 & -0.004 & -0.018 \\
\hline 5 & -1.26 & $\begin{array}{c}-0.49 \\
-1\end{array}$ & -0.09 & -0.08 & -0.94 & 0.92 & -0.35 & -0.08 & -0.648 & -0.529 \\
\hline 6 & 0.29 & -0.08 & 0.13 & 0.13 & 0.45 & 0.98 & 0.04 & 0.06 & 0.388 & 0.332 \\
\hline 7 & 0.04 & 0.12 & 0.02 & -0.01 & 0.69 & 0.33 & 0.08 & 0.02 & -0.056 & 0.055 \\
\hline 8 & 0.49 & -0.11 & 0.08 & 0.09 & 0.31 & 0.12 & 0.08 & 0.02 & 0.424 & 0.362 \\
\hline 9 & 0.79 & 0.63 & 0.00 & 0.03 & -0.77 & 0.50 & 0.19 & -0.03 & -0.303 & -0.276 \\
\hline 1 ' & -0.98 & 0.36 & -0.09 & -0.10 & 0.19 & -0.85 & 0.45 & -0.06 & -0.235 & -0.217 \\
\hline 2 & -0.26 & -0.10 & 0.02 & 0.01 & -0.76 & 1.18 & -0.58 & 0.01 & -0.307 & -0.185 \\
\hline 3 , & 0.88 & -0.47 & 0.01 & 0.01 & -0.65 & 0.84 & -0.33 & -0.05 & -0.095 & -0.087 \\
\hline 4 ' & -0.04 & -0.67 & -0.02 & 0.01 & 0.57 & 0.21 & 0.15 & -0.03 & 0.226 & 0.097 \\
\hline 5 , & 0.41 & 0.89 & 0.08 & 0.08 & 0.65 & -1.37 & 0.32 & 0.13 & 0.411 & 0.393 \\
\hline \multirow[t]{2}{*}{$\mathrm{A} / \mathrm{s}_{\mathrm{ij}}$} & -1.37 & -1.56 & -0.12 & -0.15 & -2.53 & -2.31 & -1.07 & -0.24 & -1.003 & -0.844 \\
\hline & 1.48 & 1.36 & 0.14 & 0.14 & 1.68 & 1.86 & 0.71 & 0.22 & 1.472 & 1.286 \\
\hline \multirow[t]{2}{*}{$\mathrm{m}_{\mathrm{H}}$} & 58.9 & 25.5 & 2.20 & 1.28 & 37.9 & 14.0 & 16.1 & 4.55 & 8.973 & 7.611 \\
\hline & \multicolumn{10}{|c|}{ 2nd crop (safrinha) } \\
\hline 1 & -0.30 & 0.23 & 0.00 & -0.06 & -0.55 & -0.26 & 0.18 & -0.08 & -0.225 & 0.207 \\
\hline 2 & 0.20 & 0.16 & -0.05 & -0.04 & -0.15 & 0.14 & -0.16 & -0.02 & -0.006 & 0.029 \\
\hline 3 & -0.05 & 0.07 & -0.02 & -0.04 & 0.35 & -0.06 & -0.20 & -0.03 & -0.043 & -0.081 \\
\hline 4 & 0.15 & 0.32 & -0.03 & -0.02 & -0.07 & -0.16 & 0.11 & -0.01 & -0.008 & 0.009 \\
\hline 5 & $\begin{array}{l}-0.60 \\
\end{array}$ & -1.33 & -0.09 & -0.08 & -1.10 & -1.26 & $\begin{array}{l}-0.49 \\
\end{array}$ & -0.09 & -0.638 & -0.577 \\
\hline 6 & 0.45 & 0.74 & 0.09 & 0.12 & -0.01 & 0.29 & -0.08 & 0.13 & 0.400 & 0.349 \\
\hline 7 & 0.35 & -0.32 & 0.01 & -0.01 & 1.50 & 0.04 & 0.12 & 0.02 & -0.191 & 0.158 \\
\hline 8 & -0.05 & 0.17 & 0.09 & 0.11 & 1.02 & 0.49 & -0.11 & 0.08 & 0.182 & 0.177 \\
\hline 9 & -0.15 & $\begin{array}{l}-0.04 \\
\end{array}$ & 0.00 & 0.02 & $\begin{array}{l}-0.99 \\
\end{array}$ & 0.79 & 0.63 & 0.00 & -0.332 & -0.270 \\
\hline 1' & -0.58 & -0.20 & -0.09 & 0.11 & -0.18 & -0.98 & 0.36 & -0.09 & -0.133 & -0.116 \\
\hline 2 ' & -0.42 & 0.06 & 0.00 & -0.07 & 0.59 & -0.26 & -0.10 & 0.02 & -0.196 & -0.133 \\
\hline 3 ' & 0.39 & -0.25 & 0.05 & -0.03 & -0.42 & 0.88 & -0.47 & 0.01 & 0.264 & 0.246 \\
\hline 4 ' & 0.08 & -0.60 & -0.04 & 0.14 & -0.16 & -0.04 & -0.67 & -0.02 & 0.138 & -0.194 \\
\hline 5 , & 0.53 & 1.00 & 0.08 & 0.05 & 0.17 & 0.41 & 0.89 & 0.08 & 0.203 & 0.270 \\
\hline $\mathrm{A} / \mathrm{sij}_{\mathrm{ij}}$ & -1.04 & -1.28 & -0.13 & -0.11 & -1.62 & -2.51 & -1.95 & -0.23 & -0.585 & -0.504 \\
\hline & 1.18 & 1.49 & 0.12 & 0.10 & 1.18 & 2.15 & 1.31 & 0.26 & 0.652 & 0.558 \\
\hline $\mathrm{m}_{\mathrm{H}}$ & 60.6 & 24.1 & 2.22 & 1.26 & 36.7 & 12.6 & 14.2 & 4.11 & 6.927 & 5.848 \\
\hline
\end{tabular}

For abbreviations, see Tables 1 or $2 . \mathrm{A} / \mathrm{s}_{\mathrm{ij}}=$ range for estimates of specific combining ability effects.

The significance of GCA for the traits directly associates with yield (EY and GY) assures a good perspective for the selection process herein proposed, and before looking to this topic it is readily seen the outstanding parent varieties as indicated by their high and positive effects of GCA; in fact, for GY the higher estimates of $g_{i}$ and $g_{j}$ were shown by NAP8 in Group I and by HG-5 Group II in the 1st crop; in the 2nd crop the higher estimates were for NAP-6 and HG-5, respectively.

\section{Prediction of population means}

As already shown, the main focus of the present study is directed to the synthesis of new populations by exploiting exotic germplasm in combination with local and adapted populations. In this sense, two types of populations are to be developed: a) Population resulting from the recombination of the variety cross (NAP x HG = Group I x Group II). b) Hybrid combinations (NAP x HG) most appropriate for the exploitation of heterosis at 
the interpopulation level. By using the procedure already presented, the predicted means of populations identified in (a) are shown in Table 12, for the highest yielding combinations; the predicted means were obtained by first identifying the varieties with positive effects of GCA for grain yield and the prediction procedure was accomplished based only in the selected varieties. Then, the predicted means (Table 12) represent the best combination for each variety of Group I.

\begin{tabular}{|c|c|c|c|c|c|c|c|c|c|}
\hline $\mathrm{P}_{\mathrm{ij}}$ & GY & MF & SD & $\mathrm{PH}$ & EH & TL & NB & EL & ED \\
\hline \multicolumn{10}{|c|}{1 st crop (safra) - season $\varepsilon_{1}$} \\
\hline $1 \times 5^{\prime}$ & 8.47 & 59.3 & 26.6 & 2.29 & 1.30 & 39.1 & 11.8 & 16.3 & 4.80 \\
\hline $2 \times 5^{\prime}$ & 8.20 & 59.1 & 26.3 & 2.25 & 1.33 & 38.6 & 12.4 & 16.6 & 4.78 \\
\hline $3 \times 55^{\prime}$ & 8.43 & 59.3 & 26.3 & 2.27 & 1.29 & 39.7 & 12.5 & 16.4 & 4.67 \\
\hline $4 \times 4{ }^{\prime}$ & 8.01 & 58.8 & 24.7 & 2.24 & 1.32 & 39.2 & 12.7 & 17.1 & 4.63 \\
\hline $5 \times 3^{\prime}$ & 7.55 & 58.6 & 25.7 & 2.27 & 1.33 & 37.6 & 15.3 & 15.7 & 4.56 \\
\hline $6 \times 5^{\prime}$ & 8.43 & 59.8 & 25.8 & 2.41 & 1.47 & 39.2 & 13.2 & 16.3 & 4.83 \\
\hline $7 \times 3^{\prime}$ & 8.86 & 60.0 & 25.5 & 2.36 & 1.39 & 38.5 & 14.8 & 16.5 & 4.59 \\
\hline $8 \times 4$ & 8.30 & 58.6 & 25.4 & 2.35 & 1.44 & 39.7 & 13.7 & 17.3 & 4.69 \\
\hline $9 \times 55^{\prime}$ & 7.86 & 59.7 & 26.4 & 2.35 & 1.41 & 37.8 & 12.6 & 16.7 & 4.70 \\
\hline \multicolumn{10}{|c|}{ 2nd crop (safrinha) - season } \\
\hline $1 \times 3^{\prime}$ & 5.48 & 60.7 & 24.8 & 2.32 & 1.31 & 38.8 & 13.2 & 14.2 & 4.28 \\
\hline $2 \times 3^{\prime}$ & 5.73 & 60.4 & 23.8 & 2.28 & 1.31 & 38.2 & 11.7 & 15.0 & 4.28 \\
\hline $3 \times 3^{\prime}$ & 5.26 & 60.9 & 23.5 & 2.28 & 1.31 & 36.6 & 12.0 & 15.0 & 4.18 \\
\hline $4 \times 3^{\prime}$ & 5.66 & 60.6 & 24.2 & 2.34 & 1.32 & 38.0 & 13.7 & 15.0 & 4.20 \\
\hline $5 \times 3^{\prime}$ & 5.13 & 59.8 & 22.8 & 2.31 & 1.31 & 36.6 & 13.5 & 14.2 & 4.13 \\
\hline $6 \times 5^{\prime}$ & 6.39 & 61.0 & 26.1 & 2.43 & 1.41 & 38.3 & 12.3 & 14.9 & 4.23 \\
\hline $7 \times 3^{\prime}$ & 6.05 & 60.8 & 23.9 & 2.42 & 1.41 & 37.2 & 13.5 & 15.0 & 4.23 \\
\hline $8 \times 4^{\prime}$ & 5.77 & 60.1 & 24.4 & 2.36 & 1.41 & 37.0 & 13.9 & 15.1 & 4.22 \\
\hline $9 \times 3^{\prime}$ & 5.40 & 60.8 & 23.8 & 2.38 & 1.41 & 36.0 & 14.1 & 14.8 & 4.19 \\
\hline
\end{tabular}

$\mathrm{P}_{\mathrm{ij}}$ - obtained by recombination of the hybrid cross $\mathrm{H}_{\mathrm{ij}}=\mathrm{V}_{\mathrm{i}} \times \mathrm{V}_{\mathrm{j}}$. For abbreviations, see Tables 1 or 2.

In season $\mathrm{e}_{1}$, the expected mean ( $\mathrm{t} / \mathrm{ha}$ ) of the selected combinations varied from 7.550 $\left(5 \times 3^{\prime}=\right.$ NAP-HT $\times$ HG-55) to 8.860 ( 7 x $3^{\prime}=$ NAP-PZ $\times$ HG-55); the range is equivalent to 95.4 and $106.9 \%$ of the check mean. In the 2 nd crop (season $\mathrm{e}_{2}$ ) grain yield varied from 5.130 $\left(5 \times 3^{\prime}\right)$ to $6.390\left(2 \times 3^{\prime}=\right.$ NAP-FL x HG-55), which represents 97.6 and $108.8 \%$ of the check mean. Notice that HG-55 was elected, in both seasons, as the most promising parent variety of Group II to be used as the base for incorporation of two varieties of Group I. In fact, HG-55 ranked in first and second place for positive GCA in the seasons $\mathrm{e}_{1}$ and $\mathrm{e}_{2}$, respectively. The expected means for the other traits of the selected crosses are also shown in Table 12 in order to complement the amount of useful information about the germplasm in use.

The prediction of hybrid populations (composites) [Group I x Group II = crosses of populations developed from varieties of positive effects of GCA], led to the identification of the five most promising crosses between composites (or variety $\mathrm{x}$ variety, or variety $\mathrm{x}$ composite) as shown in Table 13. In season $\mathrm{e}_{1}$, the expected GYs ( $\mathrm{t} / \mathrm{ha}$ ) were very similar among crosses, in the range of 8.273 to 8.428 , equivalent to 92.0 and $93.7 \%$ of the hybrid check. In season $\mathrm{e}_{2}$ the range was from 6.021 to 6.392 , representing 95.2 and $101.1 \%$ of the hybrid check. The expected pattern of expression of the other traits is also shown in Table 13. Taking into account that the number of populations is relatively low, it should be advisable the synthesis of the new parental populations and the evaluation of the crosses between populations. For season $\mathrm{e}_{1}$, from Group I the set includes three open-pollinated varieties $(3,6,8)$, one composite of size 2 (36) and one composite of size 3 (368); from Group II, it includes two open-pollinated

Genetics and Molecular Research 16 (1): gmr16019544 
Table 13. Expected grain yield and eight plant and ear traits of the five highest yielding crosses between composites from two groups of populations in two crop seasons.

\begin{tabular}{|c|c|c|c|c|c|c|c|c|c|}
\hline Cross & GY & MF & SD & $\mathrm{PH}$ & EH & TL & NB & EL & ED \\
\hline \multicolumn{10}{|c|}{ 1st crop (safra) } \\
\hline$(368) \times(5)$ & 8.273 & 59.7 & 26.6 & 2.47 & 1.49 & 40.4 & 13.3 & 16.9 & 4.85 \\
\hline$(8) \times(4)$ & 8.302 & 59.0 & 25.6 & 2.51 & 1.59 & 40.6 & 14.4 & 17.4 & 4.85 \\
\hline (3) x (5) & 8.427 & 59.5 & 27.0 & 2.41 & 1.40 & 41.1 & 13.0 & 17.7 & 4.75 \\
\hline$(36) \times(5)$ & 8.427 & 59.6 & 26.6 & 2.50 & 1.51 & 40.6 & 13.2 & 17.2 & 4.85 \\
\hline$(6) \times(5)$ & 8.428 & 59.8 & 26.2 & 2.58 & 1.61 & 40.1 & 13.4 & 16.7 & 4.95 \\
\hline \multicolumn{10}{|c|}{ 2nd crop (safrinha) } \\
\hline$(67) \times(5)$ & 6.021 & 60.6 & 26.1 & 2.50 & 1.46 & 37.0 & 11.4 & 15.4 & 4.40 \\
\hline$(6) \times(35)$ & 6.035 & 61.3 & 26.0 & 2.53 & 1.51 & 38.3 & 14.7 & 16.1 & 4.45 \\
\hline (7) $\times(3)$ & 6.046 & 61.0 & 24.6 & 2.52 & 1.43 & 37.8 & 12.2 & 16.1 & 4.25 \\
\hline$(16) \times(5)$ & 6.162 & 59.9 & 25.7 & 2.46 & 1.41 & 36.5 & 12.2 & 16.6 & 4.43 \\
\hline$(6) \times(5)$ & 6.392 & 60.5 & 26.8 & 2.56 & 1.52 & 38.3 & 12.5 & 15.8 & 4.45 \\
\hline
\end{tabular}

For abbreviations, see Tables 1 or 2.

varieties $(4,5)$. For the season $e_{2}$, it comprises two varieties $(6,7)$ and two composites $(16$ and 67) from Group I, while from Group II it includes two varieties $(3,5)$ and one composite (35). The next step is to accomplish the crosses following the schedule shown in Table 13.

\section{ACKNOWLEDGMENTS}

Research supported by Fundação de Apoio à Pesquisa do Estado de Goiás (FAPEG).

\section{REFERENCES}

Andrade JAC and Miranda Filho JB (2008). Quantitative variation in the tropical maize population, ESALQ-PB1. Sci. Agric. 65: 174-182. http://dx.doi.org/10.1590/S0103-90162008000200011

Basso CW and Miranda Filho JB (2001). Médias preditas de produção de compostos de milho sob severa epidemia de enfezamento no sudeste do Brasil. Summa Phytopathol. 27: 23-30.

CONAB (Companhia Nacional de Abastecimento) (2015). Acompanhamento de safra brasileira: grãos, quarto levantamento, maio 2015 - Brasília: Conab.

Eberhart SA, Harrison MN and Ogada FA (1967). A comprehensives breeding system. Der Zuchter 37: 169-174.

Gardner CO and Eberhart SA (1966). Analysis and interpretation of the variety cross diallel and related populations. Biometric. 22: 439-452. http://dx.doi.org/10.2307/2528181

Geraldi IO and Miranda Filho JB (1988). Adapted models for the analysis of combining ability of varieties in partial diallel crosses. Braz. J. Genet. 11: 419-430.

Goodman MM (1985). Exotic maize germplasm: status and prospects. Iowa State J. Res. 59: 497-527.

Griffing B (1956). Concept of general and specific combining ability in relation to diallel crossing systems. Aust. J. Biol. Sci. 9: 463-493. http://dx.doi.org/10.1071/BI9560463

Hallauer AR and Miranda Filho JB (1988). Quantitative genetics in maize breeding. Iowa State University Press, Ames, 468.

Jenkins MT (1934). Methods of estimating the performance of double crosses in corn. J. Am. Soc. Agron. 26: 199-204. http://dx.doi.org/10.2134/agronj1934.00021962002600030004x

Jenkins MT (1978). Maize breeding during the development and early years of hybrid maize. In: Maize Breeding and Genetics (Walden DB, ed.) John Wiley, New York, 13-28.

Mather K and Jinks JL (1982). Biometrical Genetics. Chapman and Hall, London.

Mendes UC, Miranda Filho JB, Oliveira AS and Reis EF (2015). Heterosis and combining ability in crosses between two groups of open-pollinated maize populations. Crop Breed. Appl. Biotechnol. 15: 235-243. http://dx.doi. org/10.1590/1984-70332015v15n4a40

Miranda Filho JB (1974). Cruzamentos dialélicos e síntese de compostos com ênfase na produtividade e no porte da planta. Tese (Doutorado) - Escola Superior de Agricultura “Luiz de Queiroz", Universidade de São Paulo, Piracicaba.

Miranda Filho JB (1992). Exotic Germoplasms Introduced in a Brazilian Maize Breeding. Braz. J. Genet. 15: 631-642.

Genetics and Molecular Research 16 (1): gmr16019544 
Miranda Filho JB and Chaves LJ (1991). Procedures for selecting composites based on prediction methods. Theor. Appl. Genet. 81: 265-271. http://dx.doi.org/10.1007/BF00215732

Miranda Filho JB and Geraldi IO (1984). An adapted model for the analysis of partial diallel crosses. Braz. J. Genet. 7: 677-688.

Miranda Filho JB and Viégas GP (1987). Milho híbrido. In: Paterniani E and Viégas GP. Melhoramento e produção de milho. Volume I. Campinas: Fundação Cargill, p. 277-340.

Miranda Filho JB, Nass LL, Santos MX and Regitano Neto A (2000). Avaliação de acessos de milho para resistência a doenças foliares. Embrapa Recursos Genéticos e Biotecnologia, Brasília, 147p. (Circular Técnica, 3).

Moro JR, Naspolini Filho V, Viana RT and Gama EEG (1981). Introdução de novos germoplasmas de milho no Brasil. Pesqui. Agropecu. Bras. 16: 867-882.

Nass LL, Miranda Filho JB and Santos MX (2001). Uso de Germoplasma Exótico no Melhoramento. In: Recursos Genéticos e Melhoramento - Plantas (Nass LL, Valois ACC, Melo IS and Valadares-Inglis MC, eds.). Fundação MT, Rondonópolis, 101-122.

Oliveira AS, Miranda Filho JB and Reis EF (2015). Variability and inbreeding in semiexotic maize populations. Genet. Mol. Res. 14: 1184-1199. http://dx.doi.org/10.4238/2015.February.6.21

Regitano Neto A, Nass LL and Miranda Filho JB (1997). Potential of twenty exotic germplasms to improve Brazilian maize architecture. Braz. J. Genet. 20: 691-696.

Santos MX and Miranda Filho JB (1992). Genetic potential of two Brazilian races of maize for breeding purposes. $J$. Genet. Breed. 46: 83-90.

Souza AC (2015). Variabilidade genética em três populações de milho. Dissertação (Mestrado em Agronomia - Produção Vegetal) - Universidade Federal de Goiás, Regional Jataí, Jataí.

Sprague GF and Tatum LA (1942). General vs. specific combining ability in single crosses of corn. J. Am. Soc. Agron. 34: 923-932. http://dx.doi.org/10.2134/agronj1942.00021962003400100008x

Vencovsky R and Barriga P (1992). Genética biométrica no fitomelhoramento. Sociedade Brasileira de Genética, Ribeirão Preto, 496p. 